\title{
Prefrontal Neurons Represent Winning and Losing during Competitive Video Shooting Games between Monkeys
}

\author{
Takayuki Hosokawa ${ }^{1,2}$ and Masataka Watanabe ${ }^{1}$ \\ ${ }^{1}$ Department of Physiological Psychology, Tokyo Metropolitan Institute of Medical Science, Setagaya, Tokyo, 156-8506, Japan, and ${ }^{2} \mathrm{Helen}$ Wills \\ Neuroscience Institute, University of California at Berkeley, Berkeley, California 94720-3190
}

Humans and animals must work to support their survival and reproductive needs. Because resources are limited in the natural environment, competition is inevitable, and competing successfully is vitally important. However, the neuronal mechanisms of competitive behavior are poorly studied. We examined whether neurons in the lateral prefrontal cortex (LPFC) showed response sensitivity related to a competitive game. In this study, monkeys played a video shooting game, either competing with another monkey or the computer, or playing alone without a rival. Monkeys performed more quickly and more accurately in the competitive than in the noncompetitive games, indicating that they were more motivated in the competitive than in the noncompetitive games. LPFC neurons showed differential activity between the competitive and noncompetitive games showing winning- and losing-related activity. Furthermore, activities of prefrontal neurons differed depending on whether the competition was between monkeys or between the monkey and the computer. These results indicate that LPFC neurons may play an important role in monitoring the outcome of competition and enabling animals to adapt their behavior to increase their chances of obtaining a reward in a socially interactive environment.

\section{Introduction}

Competition is a crucial part of the lives of both animals and humans. In nature, animals typically compete against each other to obtain limited resources, such as food and mates. Winning a competition improves, and losing diminishes, chances of survival and reproduction. Humans typically face competitive situations, including contests, sports, and business. Winning may improve, whereas losing may degrade, one's social status. Thus, winning competitions is vitally important in our lives. Although there are many psychological and sociological studies on competition (Reeve et al., 1985; Washburn et al., 1990; Garcia et al., 2006), the neuronal mechanisms of competitive behavior are poorly studied. Because competitions occur predominantly in social situations in which there are opponents, social factors are important in competition. Although there are neurophysiological studies that examined neuronal activities of several brain areas while monkeys performed tasks simulating competitive games, they

Received Dec. 25, 2011; revised April 18, 2012; accepted April 18, 2012.

Author contributions: T.H. and M.W. designed research; T.H. performed research; T.H. analyzed data; T.H. and M.W. wrote the paper.

This study was supported by a grant from the Nakayama Foundation for Human Science (T.H.), a grant from the Hayao Nakayama Foundation (M.W.), Japan Society for the Promotion of Science Grant-in-Aid for JSPS Fellows $09 J 07528$ (T.H.), and Grant-in-Aid for Scientific Research on Priority Areas (System Study on Higher-Order Brain Functions) 17022050, Grant-in-Aid for Scientific Research (B) 22330205, and Grant-in-Aid for Scientific Research on Innovative Areas (Neural Creativity for Communication) 22120524 from the Ministry of Education Culture, Sports, Science, and Technology of Japan (M.W.) This study was also supported by the National BioResource Project "Japanese Monkeys" of the Ministry of Education Culture, Sports, Science, and Technology of Japan. We thank W. Schultz, M. Sakagami, and K. Tsutsui for helpful comments on a previous version of this manuscript.

The authors declare no competing financial interests.

Correspondence should be addressed to Masataka Watanabe, Department of Physiological Psychology, Tokyo Metropolitan Institute of Medical Science, Kamikitazawa 2-1-6, Setagaya, Tokyo, 156-8506, Japan. E-mail: watanabe-ms@igakuken.or.jp.

DOI:10.1523/JNEUROSCI.6479-11.2012

Copyright $\odot 2012$ the authors $\quad 0270-6474 / 12 / 327662-10 \$ 15.00 / 0$ were conducted without social interactions, i.e., with only one monkey playing a game against an inanimate computer (Barraclough et al., 2004; Dorris and Glimcher, 2004; Seo and Lee, 2007; Thevarajah et al., 2009). Thus, we aimed to study neuronal activity related to competitive behavior and to compare competition-related neuronal activity between social and nonsocial conditions.

We trained monkeys to play competitive and noncompetitive games. As previous behavioral studies showed (Rumbaugh et al., 1989; Washburn et al., 1990), monkeys were able to play video shooting games by manipulating joysticks to shoot bullets at a target. We had two types of competitive games: monkey-monkey (Mon-Mon) and monkey-computer (Mon-Com) competitions. Two monkeys competed against each other in the MonMon competition (social condition; see Fig. $1 \mathrm{~A}$ ), whereas one monkey competed against an inanimate computer in the MonCom competition (nonsocial condition; see Fig. $1 B$ ). In the noncompetitive game, a monkey played the game without a rival, in which no bullets came from the other side (see Fig. 1C). We recorded neuronal activities while the monkeys played these video games.

We were especially interested in the lateral prefrontal cortex (LPFC), because competition involves both cognitive (such as rules and strategy) and motivational (desire to obtain a reward) components, and the LPFC is known to be involved in the integration of cognitive and motivational information (Kobayashi et al., 2002; Watanabe and Sakagami, 2007; Pan et al., 2008). Furthermore, the LPFC is also involved in the processing of social information (Zink et al., 2008; Fujii et al., 2009). We hypothesized that activities of LPFC neurons would differ between the competitive and noncompetitive situations and also differentiate the competitive situation in which the rival was another monkey 


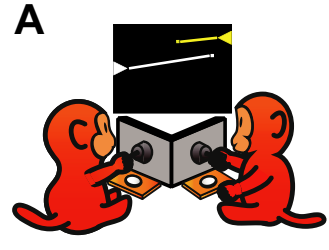

D

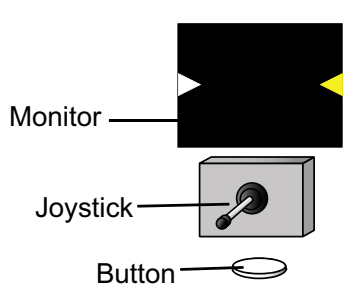

F
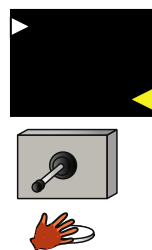

$$
\text { Start }
$$

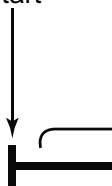

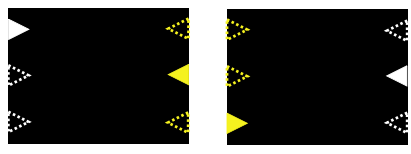

E 政

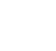

A

B
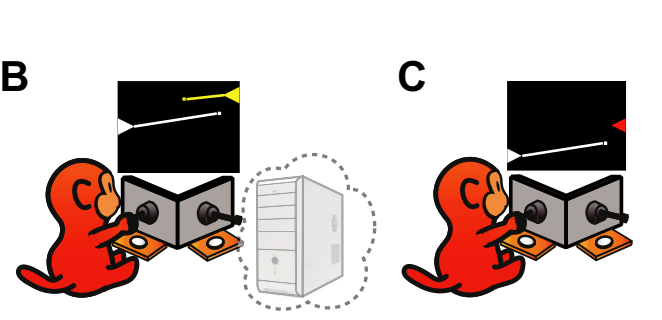

game. Before participating in this study, the monkeys had been trained in other tasks and were accustomed to manipulating a joystick. They learned to play these games in $\sim 3$ months. When neuronal recordings started, the monkeys had been well trained in these tasks for $>9$ months. The experimental tasks were controlled by a TEMPO system (Reflective Computing). The experiments were conducted in a dimly lit sound-attenuated booth.

(1) In the Mon-Mon competitive game, two monkeys competed against each other. Two monkeys faced a computer monitor (LCDAD193VB; I-O Data), arranged at an angle so that they could see each other (Fig. 1A). A horizontally protruding joystick (40JBK-YO20R2G; Sakae Tsushin Kogyo) and a button (OBSA-60UM; Sanwa Denshi) were in front of each monkey (Fig. 1D). When both of the monkeys pushed their own button, a trial started and two colored (white and yellow) triangles appeared on the left and right sides of the monitor, facing each other (Fig. $1 E$ ). The triangle represented a turret, and a bullet was launched from the triangle in the direction that the joystick was tilted. The monkeys had to return the joystick to the neutral position to shoot another bullet. The trajectory of a bullet was linear and could not be changed after it was launched. We trained the monkeys to tilt the joystick and shoot at the turret (target) on the other side. Once the monkey shot a bullet, another bullet could not be launched until the bullet disappeared from view. The color of the turret was fixed for each monkey: white for monkeys $\mathrm{H}$ and $\mathrm{S}$, and yellow for monkey P. The positions of the turrets were randomly selected from top, middle, or bottom, and left or right (Fig. $1 E$ ). These positions changed from trial to trial, but they were fixed within a trial. The monkey that made the first successful hit was the winner. A drop of grape juice $(0.3 \mathrm{ml})$ was delivered to the winner from a spout positioned in front of the monkey's mouth via a solenoid valve that emitted an audible click at the time of its operation. The loser did not receive any reward. When a monkey hit the target, a $1 \mathrm{~s}$ beep was presented, followed (in the case of the winner) or not followed (in the case of the loser) by a reward. The beep was always

from that in which the rival was an inanimate computer. We found that LPFC neurons were sensitive to the competition and animacy of the rival, with most of them showing enhanced activity in the competitive game, especially when the rival was another monkey. The results indicate the importance of LPFC in competitive situations to increase chances of winning.

\section{Materials and Methods}

Animal subjects. We used three Japanese macaque monkeys (Macaca fuscata; monkey H: male, $7.1 \mathrm{~kg}$; monkey S: female, $6.4 \mathrm{~kg}$; and monkey P: male, $8.2 \mathrm{~kg}$ ). All experiments were conducted in accordance with the National Academies Press guidelines for animal experiments and were approved by the ethics committee of our institute. During the experiments, which were conducted on weekdays, the monkeys obtained all of their fluids by playing games, but they were given ad libitum access to water during the weekend.

Types of shooting games. We trained monkeys to play (1) Mon-Mon competitive, (2) Mon-Com competitive, and (3) noncompetitive video shooting games, as well as (4) a modified version of noncompetitive the same regardless of which monkey won the game. During the beep, a white cross appeared on the monitor (Fig. $1 F$ ) in the trials when the white-turret monkey won the game, and a yellow cross appeared when the yellow-turret monkey won. The monkeys had to push the button to advance to the next trial after the competition came to an end (after a bullet hit the target). To ensure that both monkeys were motivated to play the game, the next trial did not start until both monkeys pushed their own buttons. The intertrial interval differed depending on the monkey's response time but was set to be $>2 \mathrm{~s}$.

(2) In the Mon-Com competitive game, the rival was a computer. This game was the same as the Mon-Mon competition, except that the rival was not another monkey but an inanimate computer. Here, only one monkey played the game in the experimental booth (Fig. $1 B$ ). When the computer won the game, the solenoid valve at the winner's side operated (emitting an audible click) and the juice was delivered into a reservoir.

(3) The noncompetitive game (One-monkey noncompetitive game) was similar to the Mon-Mon competitive game, except that only one monkey played the game without a rival (Fig. 1C). When the monkey pushed the button, two colored triangles appeared on the left and right 
sides of the monitor, facing each other. The color of the target triangle was always red, and the color of the turret triangle was the monkey's own trained color, so that the monkey could recognize which side of the triangle was the target. The monkey tried to hit the target. No bullets came from the other side. In this condition, the monkey was randomly rewarded in half of the trials. It was impossible for the monkey to lose the game, and the monkey could shoot bullets until it hit the target. When the monkey hit the target, a $1 \mathrm{~s}$ beep was presented, which was followed by a reward $50 \%$ of the time. The beep was always the same regardless of the presence or absence of the reward. For the white-turret monkey, white and yellow crosses appeared during the beep in reward and no-reward trials, respectively; for the yellow-turret monkey, yellow and white crosses appeared on the monitor. Therefore, in both the competitive and noncompetitive conditions, the presentation of the monkey's own colored cross meant that the reward was going to be delivered, whereas the presentation of the cross with the other monkey's color meant that there would be no reward.

(4) The Two-monkey noncompetitive game was the same as the Onemonkey noncompetitive game, except that there was another monkey near the game-playing monkey. The other monkey just sat in the same position as in the competitive condition but did not participate in the game (see Fig. 6A-4).

Modification of reward probability during the competitive game. The reward probability was fixed at $50 \%$ in the noncompetitive conditions, whereas it differed depending on the winning rate of the monkey in the competitive conditions. To exclude the factor of reward probability in the comparison of neuronal activity between the competitive and noncompetitive conditions, we ensured that the winning rate of each monkey during the competitive conditions was close to $50 \%$. When the winning rate was unequal between the monkeys in the Mon-Mon competition condition, we gave an advantage to the weaker monkey by increasing the bullet speed. In the Mon-Com competition condition, we ensured that the monkey's winning rate would be $\sim 50 \%$ by adjusting the hitting accuracy of the computer.

Monkey's position during the training and recording periods. During the training phase, we sometimes changed the monkey's position between the left and right chairs to help them better understand that their own turret appeared on either the left or right side of the monitor, regardless of their own position. Although we also tried to train the monkeys to play the game in a reverse condition, in which the color of each monkey's turret alternated between white and yellow, we were not successful: changing the monkey's original color led to confusion.

Surgery. After the training was completed, surgery was conducted under sterile conditions. The monkey was surgically prepared under sodium pentobarbital anesthesia ( $20 \mathrm{mg} / \mathrm{kg}$ body weight, i.v.). A stainless steel recording chamber (square of $20 \times 20 \mathrm{~mm}$ ) was implanted as a microdrive receptacle on the skull stereotaxically over the prefrontal cortex. A head-restraining device ( $15 \mathrm{~mm}$ in diameter) was attached to the skull with dental acrylic. The monkey was given antibiotics every day for 1 week after the surgery.

Recordings. We conducted conventional single-neuronal recordings from the LPFC. Neuronal recordings were obtained from the monkey that was positioned on the left for technical reasons. In daily experiments, we started the recording with either the Mon-Mon competitive or Onemonkey noncompetitive condition. When we found a neuron that appeared to be task related, we recorded its activity in several other conditions. We tested the activity in the Mon-Mon competitive and noncompetitive conditions at least twice to confirm that there was no nonstationary activity change across blocks.

Neuronal activities were recorded extracellularly using tungsten electrodes $(2.0-8.0 \mathrm{M} \Omega ; \mathrm{FHC})$. An electrode was advanced with a hydraulic microdrive (MO-95C; Narishige) through a stainless steel guide tube. Neuronal activities were converted into pulses using a spike waveform detector (Multispike Detector; Alpha Omega Engineering). We recorded LPFC neuronal activity from both hemispheres of one monkey $(\mathrm{H})$ and from one hemisphere of the other monkey (S) while they were playing the games. Monkey $\mathrm{P}$ was always the competitor for both monkeys $\mathrm{H}$ and $\mathrm{S}$. Monkeys $\mathrm{H}$ and $\mathrm{S}$ never competed against each other because both of them were trained with the same turret color (white). The recording area covered both the dorsal and ventral banks of the principal sulcus (see Fig. $4 A$ ). The recording area was determined with reference to magnetic resonance images (whole-brain coverage, $2 \mathrm{~mm}$ slice thickness; Sonata 1.5T; Siemens).

We did not restrict or control the monkey's eye movements during the tasks. To examine whether there was any relationship between the competition-related neuronal activity and monkey's eye movement, we monitored the position of the monkey's eyes with an infrared eye-camera system (sampling rate, $4 \mathrm{~ms}$; R-22C-1; ISEYO Electronic). We compared the frequency of saccadic eye movements between the competitive and noncompetitive conditions during each analysis period by counting the number of saccades with amplitude exceeding $10^{\circ}$ (two-tailed MannWhitney $U$ test, $p<0.05$ ). We also compared the amount of time that the monkeys viewed different parts of the monitor. We divided the screen into nine sections $(3 \times 3)$ and calculated the amount of time that the monkeys viewed each section during each analysis period $\left(\chi^{2}\right.$ test, $p<0.05)$.

To measure licking movements, infrared photobeams and detectors were positioned across the spout. Licks were detected by breaks of an infrared beam by the monkey's tongue (sampling rate, $1 \mathrm{~ms}$ ).

Analysis of behavioral data. We used the successful hit rate of the first shot as the measure of hitting accuracy and compared it between the competitive and noncompetitive conditions. The successful hit rate of the first shot was defined as the ratio of the number of trials in which the first shot was successful to the total number of trials. In the competitive condition, the monkey was sometimes defeated before it hit the target and hence was not allowed to shoot as many bullets as in the noncompetitive condition. For this reason, we calculated the successful hit rate of the first shot, rather than that of all shots, in the comparison between the competitive and noncompetitive conditions. It should be noted that, to calculate the successful hit rate in the competitive condition, the "lose" trials in which the bullet was launched in the right direction and would have hit the target if the bullet had been shot much earlier were included as successful trials. This procedure was used to directly compare the successful hit rate of the competitive condition with that of the noncompetitive condition in which the monkey was never defeated.

\section{Results}

\section{Latency and accuracy of the first shot}

In this experiment, reward rates were made comparable between the competitive and noncompetitive tasks so that any difference in the reward rate would not affect behavior or neuronal activity between the two kinds of tasks. Thus, in the noncompetitive condition, the reward rate was fixed at 50\%. Although the reward rates could vary depending on the winning rate in the competitive conditions, we manipulated the reward rate to be close to $50 \%$ for each monkey (see Materials and Methods). The resultant reward rates were $50.8 \%$ (monkey $\mathrm{H}$ ) and $50.4 \%$ (monkey $\mathrm{S}$ ) in the Mon-Mon competition condition and $51.0 \%$ (monkey $\mathrm{H}$ ) and $50.4 \%$ (monkey S) in the Mon-Com competition condition.

We found behavioral differences in speed and accuracy between the competitive (both Mon-Mon and Mon-Com) and noncompetitive conditions. Compared with the noncompetitive condition, the latency from the start of each trial to the time of the first bullet shot was significantly shorter (Fig. 2A; Bonferroni'scorrected, two-tailed $t$ test, $p<0.05$ ), and the successful hit rate of the first shot (the ratio of the number of trials in which the first shot was successful to the total number of trials) was significantly higher (Fig. 2B; Bonferroni's-corrected, two-tailed $t$ test, $p<$ $0.05)$ during the competitive games. These findings indicate that each monkey performed more quickly and more accurately in the competitive condition and suggest that each monkey's motivation was higher in the competitive games. 
A
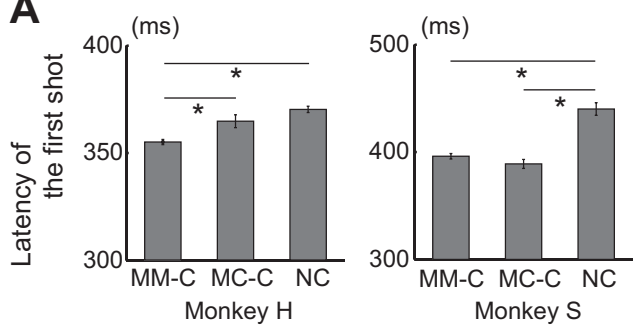

B
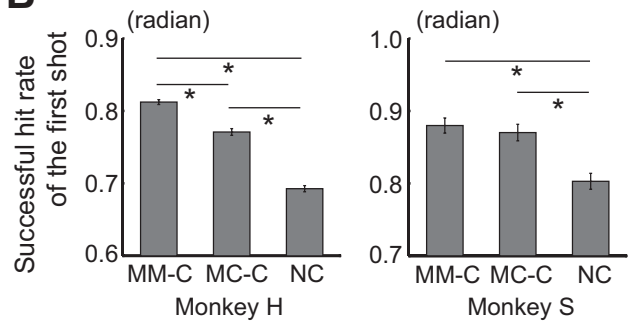

Figure 2. Behavioral data of the competitive and noncompetitive conditions. $\boldsymbol{A}$, Latency of the first shot (mean \pm SEM). Means of the median in each session were compared among the conditions. One-way ANOVA demonstrated a significant difference in latency among the three conditions (monkey H: $F_{(2,278)}=181.2, p<10^{-10}$; monkey S: $F_{(2,416)}=51.4, p<10^{-10}$ ). Post hoc paired comparisons were conducted using a Bonferroni's-corrected, two-tailed $t$ test $\left({ }^{*} p<0.05\right)$. Monkey H: $n=126$ (Mon-Mon), 29 (Mon-Com), and 126 (noncompetition). Monkey $S: n=195$ (Mon-Mon), 29 (Mon-Com), and 195 (noncompetition). $\boldsymbol{B}$, Successful hit rate of the first shot (mean $\pm S E M)$. Data were normalized by the arcsine transformation before statistical analyses. One-way ANOVA demonstrated a significant difference in the successful hit rate among the three conditions (monkey $\mathrm{H}: F_{(2,278)}=154.5, p<10^{-10}$; monkey S: $F_{(2,416)}=$ $\left.15.5, p<10^{-6}\right)$. Post hoc paired comparisons were conducted using a Bonferroni's-corrected, two-tailed $t$ test ( ${ }^{*} p<0.05$ ). Monkey H: $n=126$ (Mon-Mon), 29 (Mon-Com), and 126 (noncompetition). Monkey S: $n=195$ (Mon-Mon), 29 (Mon-Com), and 195 (noncompetition). MM-C, Mon-Mon competitive condition; MC-C, Mon-Com competitive condition; NC, noncompetitive condition.

\section{Dominant-subordinate relationship between monkeys}

We assessed the dominant-subordinate relationship between any of the two monkeys that competed against each other (monkey $\mathrm{H}$ vs $\mathrm{P}$ and monkey $\mathrm{S}$ vs $\mathrm{P}$ ). We arranged two monkeys sitting in monkey chairs face to face with a table between them. We put a food pellet on the table at approximately the same distance from each monkey, 20-30 times in a session, and counted the number of times that each monkey got the pellet. The monkeys did not hesitate to grab the pellet in the presence of the other monkey. We did not find any significant differences in the ratio of successful food retrievals between monkeys $\mathrm{H}$ and $\mathrm{P}$ (51 and 49\%, twotailed Wilcoxon's signed-rank test, $T=13.5, p=0.92, n=9$ ) or between monkeys S and P (50.4 and 49.6\%, two-tailed Wilcoxon's signed-rank test, $T=10.5, p=0.67, n=8)$. Thus, there was no clear dominant-subordinate relationship between the monkeys, at least during the dominance test used in the present study.

\section{Neuronal activities related to competition}

We recorded $321 \mathrm{LPFC}$ neurons from two monkeys (126 from monkey H; 195 from monkey S). We analyzed neuronal data in relation to winning/losing the game, as well as the presence/absence of a reward. We examined neuronal activities separately for the two periods: during the $1000 \mathrm{~ms}$ before the reward delivery (pre-reward period) and during the $1000 \mathrm{~ms}$ after the reward delivery (post-reward period; Fig. $1 F$ ). Because we found differential baseline activities (1000 ms period before the start of the trial) in many neurons (126 neurons showing higher and 26 neurons showing lower activity in the competitive compared with the noncompetitive condition, Mann-Whitney $U$ test, $p<0.05$ ), we
Table 1. Effects of competition and reward factors

\begin{tabular}{|c|c|c|c|}
\hline & Pre-reward & Post-reward & Total \\
\hline Competition and Reward factors & $66(45.5 \%)$ & $40(37.7 \%)$ & $106(42.2 \%)$ \\
\hline $\begin{array}{c}\text { competition }>\text { noncompetition and reward }> \\
\text { no-reward (Win-competitive }[+] \text { activity) }\end{array}$ & 22 & 12 & 34 \\
\hline $\begin{array}{l}\text { competition }>\text { noncompetition and } \\
\text { no-reward }>\text { reward (Lose-competitive }[+] \\
\text { activity) }\end{array}$ & 34 & 22 & 56 \\
\hline $\begin{array}{l}\text { noncompetition }>\text { competition and } \\
\text { no-reward }>\text { reward (Win-competitive }[-] \\
\text { activity) }\end{array}$ & 2 & 1 & 3 \\
\hline $\begin{array}{c}\text { noncompetition }>\text { competition and reward }> \\
\text { no-reward (Lose-competitive }[-] \text { activity) }\end{array}$ & 8 & 5 & 13 \\
\hline Competition factor & $45(31.0 \%)$ & $30(28.3 \%)$ & $75(29.9 \%)$ \\
\hline $\begin{array}{l}\text { competition }>\text { noncompetition (Win-Lose- } \\
\text { competitive }[+] \text { activity) }\end{array}$ & 38 & 23 & 61 \\
\hline $\begin{array}{l}\text { noncompetition }>\text { competition (Win-Lose- } \\
\text { competitive }[- \text { ] activity) }\end{array}$ & 7 & 7 & 14 \\
\hline Reward factor & $34(23.4 \%)$ & $36(34.0 \%)$ & $70(27.9 \%)$ \\
\hline reward $>$ no-reward & 16 & 9 & 25 \\
\hline no-reward $>$ reward & 18 & 27 & 45 \\
\hline Total & 145 & 106 & 251 \\
\hline
\end{tabular}

The number of neurons in which the main effect was significant on two-way ANCOVA (competition and reward factors) is listed.

conducted two-way ANCOVA in which activities during the baseline period served as the covariate to eliminate the possible influence of the baseline activity on pre-reward and post-reward period activity. The first factor was whether or not the condition was competitive (competition factor). The second factor was whether or not a reward was given (reward factor). In this analysis, we included only the data from the Mon-Mon competitive and One-monkey noncompetitive games. We identified 251 neurons $(78.2 \%)$ with significant effects of either one or both factors during the two analysis periods. For neurons in which significant effects were observed during both analysis periods, additional analyses were conducted on the period in which the effect size was larger. The effect size was defined as the sum of the percentage of variance explained (PVE, the ratio of the sum of squares for an independent variable to the total sum of squares) for the competition and reward factors and their interaction. Among the 251 neurons, $75(29.9 \%)$ were modulated by only the competition factor, $70(27.9 \%)$ by only the reward factor, and $106(42.2 \%)$ by both factors (Table 1). We focus here on 181 neurons ( 75 neurons with only the competition factor being significant and 106 neurons with both the competition and reward factors being significant). Hereafter, we call them "competitive neurons."

Examples of competitive neurons are illustrated in Figure 3. The neuron in Figure $3 A$ showed greater activity in reward trials under the competitive compared with the noncompetitive condition. Two-way ANCOVA revealed that both the competition and reward factors were significant (competition factor, $F_{(1,85)}=$ 28.0, $p<10^{-6}, \mathrm{PVE}=21.5 \%$; reward factor, $F_{(1,85)}=14.3, p<$ $10^{-3}, \mathrm{PVE}=11.0 \%$; interaction, $F_{(1,85)}=2.8, p=0.10, \mathrm{PVE}=$ $2.1 \%)$. The neuron in Figure $3 B$ showed a higher rate of firing in no-reward trials under the competitive compared with the noncompetitive condition. Two-way ANCOVA revealed that both the competition and reward factors, as well as interaction, were significant (competition factor, $F_{(1,105)}=10.5, p=0.002, \mathrm{PVE}=$ $7.3 \%$; reward factor, $F_{(1,105)}=21.2, p<10^{-4}$, $\mathrm{PVE}=14.8 \%$; interaction, $\left.F_{(1,105)}=4.3, p=0.04, \mathrm{PVE}=3.0 \%\right)$. The neuron in Figure $3 C$ showed higher firing rates in both reward and noreward trials under the competitive compared with the noncom- 
A Win-competitive[+] Competition Noncompetition
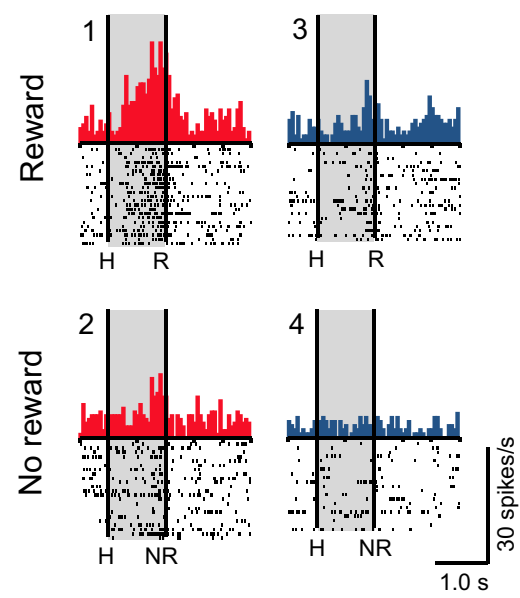

B

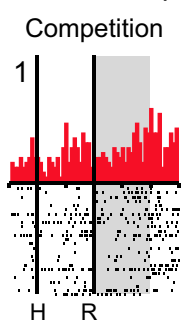

ive [+]

Noncompetition
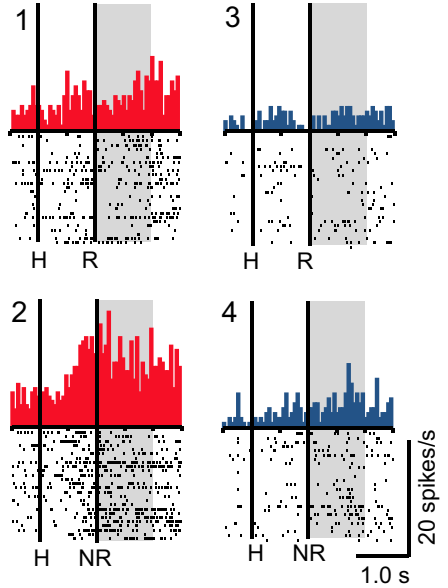

C Win-Lose-competitive[+]

Competition

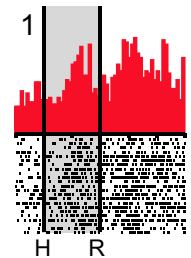

Noncompetition
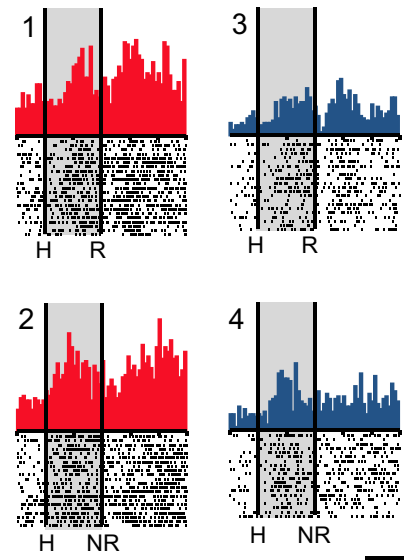

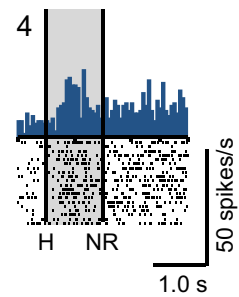

D

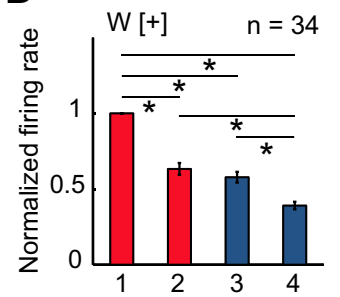

G

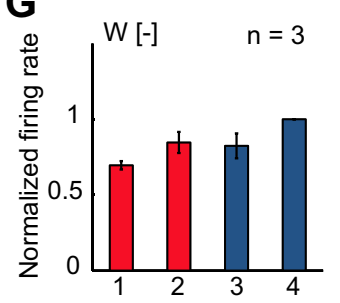

E

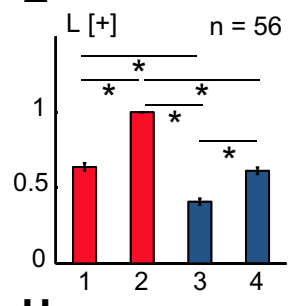

H

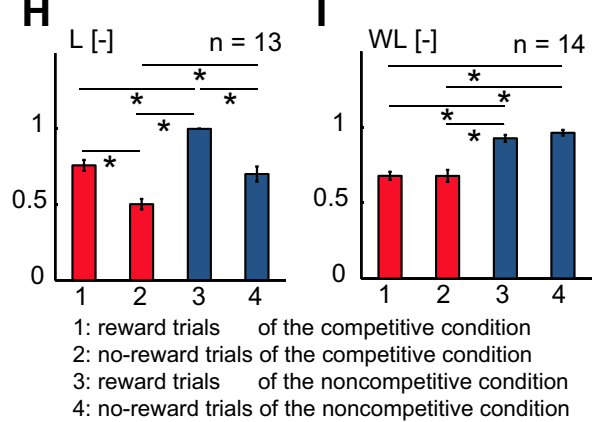

F

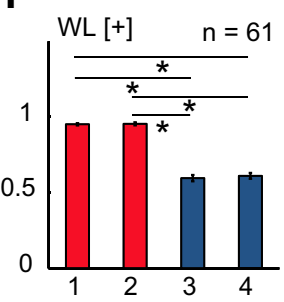

J

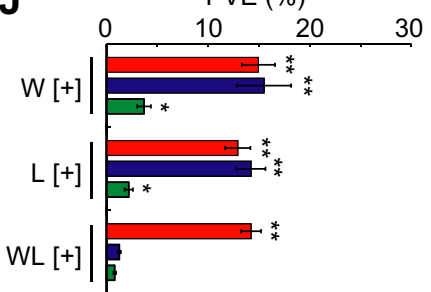

K
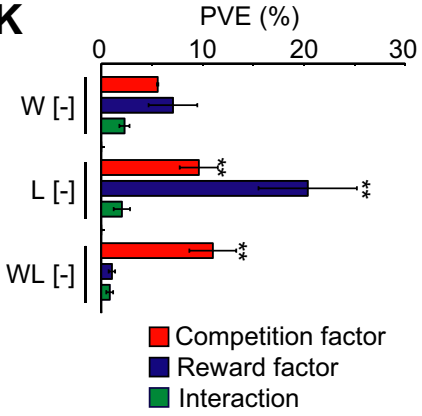

Figure 3. LPFC neurons that showed competitive activities. $\boldsymbol{A}-\boldsymbol{C}$, Raster and histogram displays for neurons that showed competitive activities: Win-competitive[+] $(\boldsymbol{A})$, Lose-competitive $[+]$ $(\boldsymbol{B})$, and Win-Lose-competitive $[+](\boldsymbol{C})$. Displays in the left and right columns show activity in the competitive and noncompetitive conditions, respectively. The top and bottom rows show activity during reward and no-reward trials, respectively. The left vertical line in each display indicates the timing of a successful hit by either monkey. The right vertical line indicates the timing of a reward delivery (reward trials) or $1 \mathrm{~s}$ after a successful hit (no-reward trials). Each shaded area indicates the period when the typical activity of each type was observed. H, Successful hit; R, reward delivery; $\mathrm{NR}, 1 \mathrm{~s}$ after a successful hit. $\boldsymbol{D}-\boldsymbol{I}$, Mean relative magnitude of activity calculated from population data for each type of neuron (normalized mean \pm SEM). $\boldsymbol{D}$, Win-competitive[+]; $\boldsymbol{E}$, Losecompetitive $[+]$; $\boldsymbol{F}$, Win-Lose-competitive[+]; $\boldsymbol{G}$, Win-competitive[-]; $\boldsymbol{H}$, Lose-competitive[-]; and $\boldsymbol{I}$, Win-Lose-competitive[-]. To obtain normalized population activity, we first obtained the mean spike rates for each neuron. We then calculated the relative magnitude of the spike rate for each type of neuron by alignment of the type of neuron in which the highest activity was observed. This analysis was conducted for the period in which the effect size was larger for each neuron. Data from different analysis periods were pooled and averaged. The mean relative magnitudes were significantly different among trial types for all types of neurons (Kruskal-Wallis $H$ test, Win-competitive $[+], H=90.46, p<10^{-19}$; Lose-competitive[ $[+], H=153.61, p<10^{-33}$; Win-Lose-competitive $[+], H=176.18, p<10^{-38}$; Win-competitive $[-], H=8.87, p=0.03$; Lose-competitive $[-], H=37.5, p<10^{-8} ;$ Win-Lose-competitive[-], $H=38.95, p<10^{-8}$ ). ${ }^{*} p<0.05$, Bonferroni's-corrected two-tailed Mann-Whitney U test. W[+], Win-competitive[+]; L[+], Lose-competitive[+]; WL[+], Win-Lose-competitive[+]; W[-], Win-competitive[ - ]; L[-], Lose-competitive[-];WL[-], Win-Lose-competitive[-].J, $\boldsymbol{K}$, Mean PVE. The plot shows the averaged PVE of each factor in the two-way ANCOVA (competition and reward factors and their interaction), separately for each type of neuron (mean \pm SEM). We used a two-tailed Mann-Whitney $U$ test to determine whether these PVE values were significantly greater than the chance level by comparing them with those calculated from the randomized data. ${ }^{*} p<0.05$; $^{* *} p<10^{-5}$.

petitive condition without differential activity between the win and lose trials under the competitive condition. Two-way ANCOVA revealed that only the competition factor was significant (competition factor, $F_{(1,83)}=23.8, p<10^{-5}$, PVE $=22.0 \%$; reward factor, $F_{(1,83)}=0.8, p=0.38, \mathrm{PVE}=0.7 \%$; interaction, $\left.F_{(1,83)}=0.4, p=0.54, \mathrm{PVE}=0.4 \%\right)$.

Among 181 competitive neurons, 151 neurons (83.4\%) showed higher and 30 neurons (16.6\%) showed lower activities in the competitive than in the noncompetitive condition. The former and latter type of neurons were designated as positive $[+]$ type and negative $[-]$ type, respectively. Higher activities in the competitive than in the noncompetitive condition (positive $[+]$ type) were observed on reward trials in 34 neurons $(18.8 \%$; Win-competitive [ + , competition $>$ noncompetition and reward $>$ no-reward; Table 1), on no-reward trials in 56 neurons (30.9\%; Lose-competitive [+], competition $>$ noncompetition and no-reward $>$ reward; Table 1$)$, and on both reward and no-reward trials in 61 neurons (33.7\%; WinLose-competitive $[+]$, competition $>$ noncompetition; Table 1). 
Among the remaining 30 neurons with lower activity in the competitive than in the noncompetitive condition (negative [-] type), lower activities were observed on reward trials in three neurons (1.7\%; Win-competitive[-], noncompetition $>$ competition and no-reward $>$ reward; Table 1 ), on no-reward trials in 13 neurons (7.2\%; Lose-competitive [-], noncompetition $>$ competition and reward $>$ no-reward; Table 1 ), and on both reward and no-reward trials in 14 neurons (7.7\%; Win-Lose-competitive[-], noncompetition $>$ competition; Table 1$)$. Figure $3 D-I$ indicates normalized population activity for Win-competitive $[+](D)$, Lose-competitive $[+](E)$, Win-Lose-competitive $[+](F)$, Win-competitive $[-](G)$, Lose-competitive $[-](H)$, and WinLose-competitive[-] $(I)$ neurons. Figure $3, J$ and $K$, indicates the mean PVE for each factor (competition factor, reward factor, and interaction) for each type of neuron. To determine whether these PVE values were significantly greater than the chance level, the PVE of each factor was compared with the PVE calculated from the randomized data combining all the competitive and noncompetitive, as well as reward and no-reward, trials for each neuron.

Locations from which these three types of neurons were recorded are illustrated in Figure 4. There was no clear segregation in any type of competitive neuron. We did not observe any difference in the frequency of saccadic eye movements or in the amount of time that the monkeys viewed each section on the monitor between the competitive and noncompetitive conditions (see Materials and Methods).

\section{Motivational effects on competitive activities}

Because the motivational level was significantly higher in the competitive than in the noncompetitive conditions (Fig. 2), it may be argued that competitive activity may simply reflect the different motivational level between the conditions but not the competitive nature of the games. To examine the possible effect of the motivational level on competitive neuronal activity, we reconducted two-way ANCOVA (competition and reward factors) on 181 competitive neurons, adding the reaction time (RT; the latency of the first shot) besides the baseline activity as a covariate. Given that the RT reflects the monkey's motivational level, we reasoned that, if competitive activities of LPFC neurons simply reflect the monkey's motivational level, then two-way ANCOVA with RT as a covariate would not reveal a significant effect of the competition factor. However, the effect of the competition factor was still significant in most of them (174 of 181), although there was no significant effect of competition in seven neurons (three Win-competitive, one Lose-competitive, and three Win-Lose-competitive).

We further analyzed the correlation between RT and neuronal activity. Because the RT reflects the animal's motivation and the LPFC is supposed to process reward expectation for the period before reward delivery and outcome evaluation after delivery, it is interesting to clarify whether the correlation between the animal's RT and activity of each type of competitive neuron is different between the pre-reward and post-reward periods. We thus calculated the Pearson's correlation coefficient between the monkey's RT and competitive neuronal activity in each neuron separately for the pre-reward and post-reward periods. The mean correlation coefficients obtained were quite small. They were within \pm 0.1 for each type of neuron in both the pre-reward and post-reward periods, except for Win-competitive $[+](-0.14 \pm$ $0.04)$ and Win-competitive $[-](0.24 \pm 0)$ (mean \pm SEM) neurons in the post-reward period. We then applied two-way ANOVA (neuron type and analysis period factors) to the correlation data separately for positive $[+]$ and negative $[-]$ type neurons and found no significant effect in any factor (positive $[+]$ type neurons, neuron type factor, $F_{(2,145)}=2.05, p=0.13$; analysis period factor, $F_{(1,145)}=0.66, p=0.42$; the interaction, $F_{(2,145)}=1.94, p=0.15$; negative $[-]$ type neurons, neuron type factor, $F_{(2,24)}=0.1, p=0.90$; analysis period factor, $F_{(1,24)}=2.28$, $p=0.14$; the interaction, $\left.F_{(2,24)}=1.68, p=0.21\right)$. Thus, there was no significant difference in RT - neuronal activity correlation between the pre-reward and post-reward periods. These results indicate that competitive activities of LPFC neurons do not reflect the monkey's RT during either the pre-reward or postreward periods and do not simply reflect the differential motivational level between the conditions.

\section{Rival's animacy and neuronal activity}

Previous neuroimaging studies have shown that human brains show differential activity depending on whether the subject believes that the rival is another human or a computer during competitive games (Gallagher et al., 2002; Sanfey et al., 2003; Rilling et al., 2004; Fukui et al., 2006). We thus examined the effect of a rival's animacy (another monkey or an inanimate computer; Fig. $1 A, B)$ on competitive neuronal activities. There were significant differences in both speed and accuracy between the Mon-Mon and Mon-Com conditions in one monkey (monkey H; Fig. $2 A, B$; Bonferroni's-corrected, two-tailed $t$ test, $p<0.05$ ), indicating that this monkey was more motivated during the MonMon competition. Furthermore, licking latency, which was defined as the latency when the monkey licked the spout for the first time after a bullet hit the target in each trial, was significantly longer when the monkeys lost a competition in the Mon-Mon than in the Mon-Com competitions in both monkeys (monkeys often made spontaneous licking movements even when there was no juice delivery) (monkey H, Mon-Mon, $554 \pm 9$ ms, MonCom, $520 \pm 9 \mathrm{~ms}$, median \pm SEM, two-tailed Mann-Whitney $U$ test, $p=0.01$; monkey S, Mon-Mon, $393 \pm 11 \mathrm{~ms}$, Mon-Com, $352 \pm 11 \mathrm{~ms}$, median \pm SEM, two-tailed Mann-Whitney $U$ test, $p=0.005)$. We analyzed 58 neurons that were recorded during both the Mon-Mon and Mon-Com competition conditions (29 neurons from monkey $\mathrm{H}, 29$ neurons from monkey S). To eliminate the possible influence of baseline activity ( 27 of the 58 neurons showed differential baseline activity between the two conditions) on pre-reward and post-reward period activity, we conducted two-way ANCOVA $(p<0.05)$ in which activities during the baseline period served as the covariate. The first factor was whether the rival was another monkey or the computer (rival factor). The second factor was whether or not a reward was given (reward factor). Forty ( 17 from monkey $\mathrm{H}$ and 23 from monkey S) of the 58 neurons showed competitive activities. Nineteen of the 40 neurons $(47.5 \%)$ were modulated by the rival factor (these 19 were all positive $[+]$ type neurons). Seventeen of them $(89.5 \%)$ showed higher and two neurons $(10.5 \%)$ showed lower activity in the Mon-Mon than in the Mon-Com competition condition. Figure $5, A$ and $B$, illustrates examples of animacy-sensitive neurons $(A$, Win-competitive $[+]$; $B$, Lose-competitive $[+])$. These neurons showed greater activity in the Mon-Mon than in the MonCom competition condition when the monkey won $(A)$ or lost $(B)$ a competition, respectively. Figure $5 C$ indicates the mean PVE of each factor (rival and reward factors and their interaction) for the 40 competitive neurons. To determine whether these PVE values were significantly greater than the chance level, the PVE of each factor was compared with the PVE calculated from the randomized data combining all the Mon-Mon and Mon-Com competitions, as well as reward and no-reward trials for each neuron. Both rival and reward factors significantly contributed to the activities of these neurons 
A

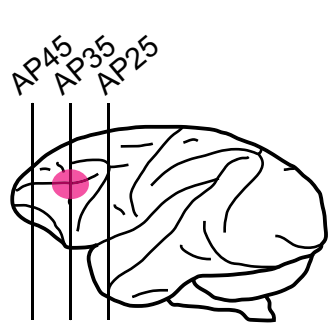

B

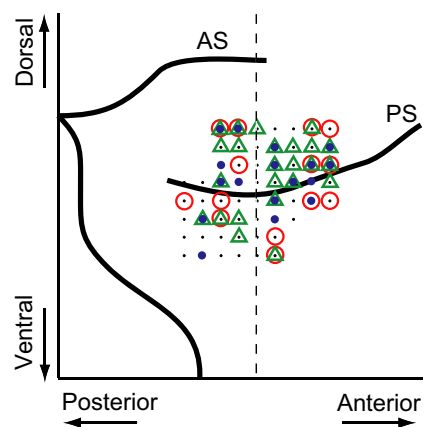

C

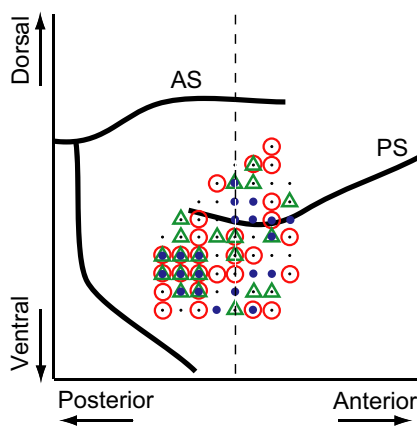

Win-competitive

- Lose-competitive

$\Delta$ Win-Lose-competitive

recording site

Figure 4. Recording sites of neurons that showed competitive activity. $\boldsymbol{A}$, Recording areas. Recording areas are highlighted by a pink ellipse on a lateral view of the monkey brain. We recorded from neurons in both the upper and lower bank of the principal sulcus. Most of the recordings were made from neurons in the region between AP30 and AP40. AP, Anterior-posterior. $\boldsymbol{B}, \boldsymbol{C}$, Recording sites of neurons that showed competitive activity were mapped onto comparative locations of the right hemisphere of each monkey's brain based on magnetic resonance images ( $\boldsymbol{B}$, monkey $\mathrm{H}$; $\boldsymbol{C}$, monkey S). The circles, dots, and triangles represent locations in which neurons showed Win-competitive (Win-competitive[+] and Win-competitive[-]), Lose-competitive (Lose-competitive[+] and Lose-competitive [-]), and Win-Lose-competitive activity (Win-Lose-competitive[+] and Win-Lose-competitive[-]), respectively. There were no biases in the recorded areas for each type of neuron across four parts separated by the dorsal and ventral parts from the principal sulcus and the anterior and posterior parts from AP 35 ( $\chi^{2}$ test; Win-activity, $\chi^{2}=2.71, p=0.44$; Lose-activity, $\chi^{2}=0.11, p=0.95$; Win-Lose-activity, $\chi^{2}=4.3, p=0.23$; the data were pooled from 2 monkeys). The vertical dashed line represents AP 35 . AS, Arcuate sulcus; PS, principal sulcus.

(Mann-Whitney $U$ test, $p<0.001)$. These results indicate that the monkey's behavior and LPFC neuronal activity were modulated by the rival's animacy.

\section{Effects of the presence of another monkey}

We examined the effect of the presence of another monkey to investigate whether competitive neuronal activity was caused by the competitive nature of the game or by the presence of another monkey within view. To do this, we introduced a modified version of the noncompetitive condition in which the monkey played the game in the presence of another monkey that was sitting nearby but did not participate in the game (Two-monkey noncompetition) (Fig. $6 A-4)$. There were no significant differences in behavioral measures of speed or accuracy between the One-monkey and Two-monkey noncompetition conditions (Fig. 6B,C; Bonferroni's-corrected, twotailed $t$ test, $p>0.05)$. Of 24 LPFC neurons that were recorded in all of the four conditions (Mon-Mon competition, Mon-Com competition, One-monkey noncompetition, and Two-monkey noncompetition, all 24 neurons were recorded from monkey $S$ ), we analyzed 20 neurons that showed competitive activity (these 20 were all positive $[+]$ type neurons) by two-way ANCOVA $(p<0.05)$. The first factor was whether or not the condition was competitive (competition factor). The second factor was whether or not there was another monkey nearby (the presence factor). We pooled the data from the reward and no-reward trials in this analysis. Fifteen neurons (75\%) were modulated by the competition factor, whereas only one neuron was modulated by the monkey's presence factor. Figure 6, D and $E$, shows examples of competitive neurons ( $D$, Win-competitive $[+]$; $E$, Lose-
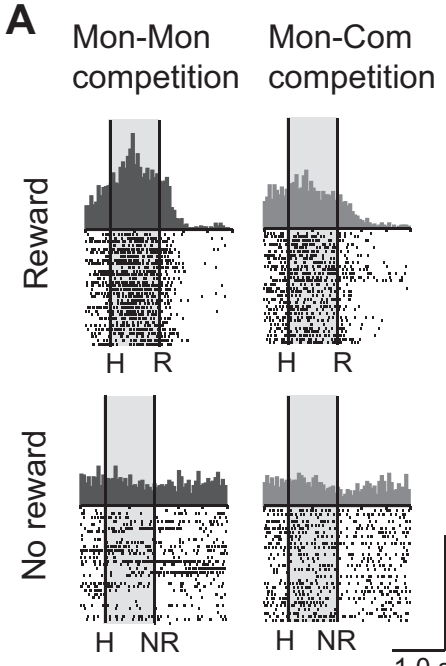
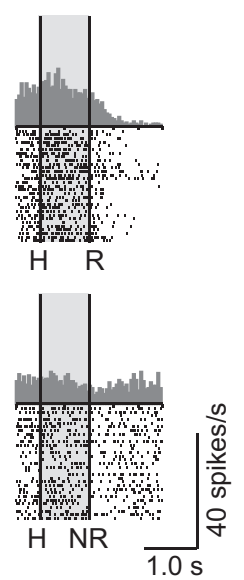

B Mon-Mon
competition
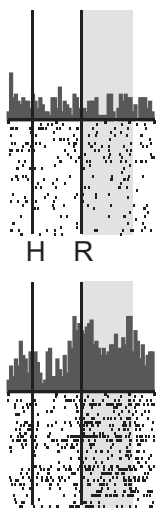

$\mathrm{H}$ NR
Mon-Com competition
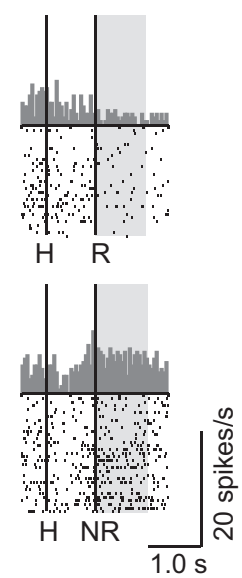

C

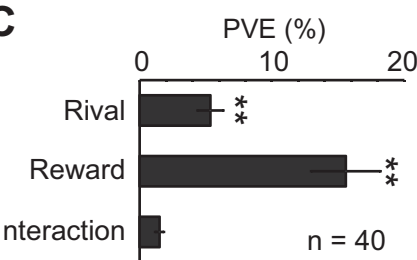

Figure 5. Effects of the rival's animacy on $L P F C$ neuronal activity. $A, B$, Examples of neurons that showed greater activity in win $(A)$ and lose $(\boldsymbol{B})$ trials during the Mon-Mon than during the Mon-Com competition. Displays in the left and right columns show activity in the Mon-Mon and Mon-Com competitions, respectively. The top and bottom rows show activity during reward and no-reward trials, respectively. Each shaded area indicates the period when typical animacy-related activity was observed. The configuration of each raster and histogram display is the same as in Figure 3A-C. C, Mean PVE for the competitive neurons that were recorded in both the Mon-Mon and Mon-Com competition conditions. The plot shows the averaged PVE of each factor in the two-way ANCOVA (rival and reward factors and their interaction) (mean \pm SEM). We used a two-tailed Mann-Whitney $U$ test to determine whether these PVE values were significantly greater than the chance level by comparing them with those calculated from the randomized data. ${ }^{* *} p<0.001$.

competitive $[+])$. These neurons showed competition-related activity, which was not modulated by the mere presence of another monkey. Figure $6 F$ indicates the mean PVE for each factor (competition and presence factors and their interaction). To determine whether these PVE values were significantly greater than the chance level, the PVE of each factor was compared with the PVE calculated 
A
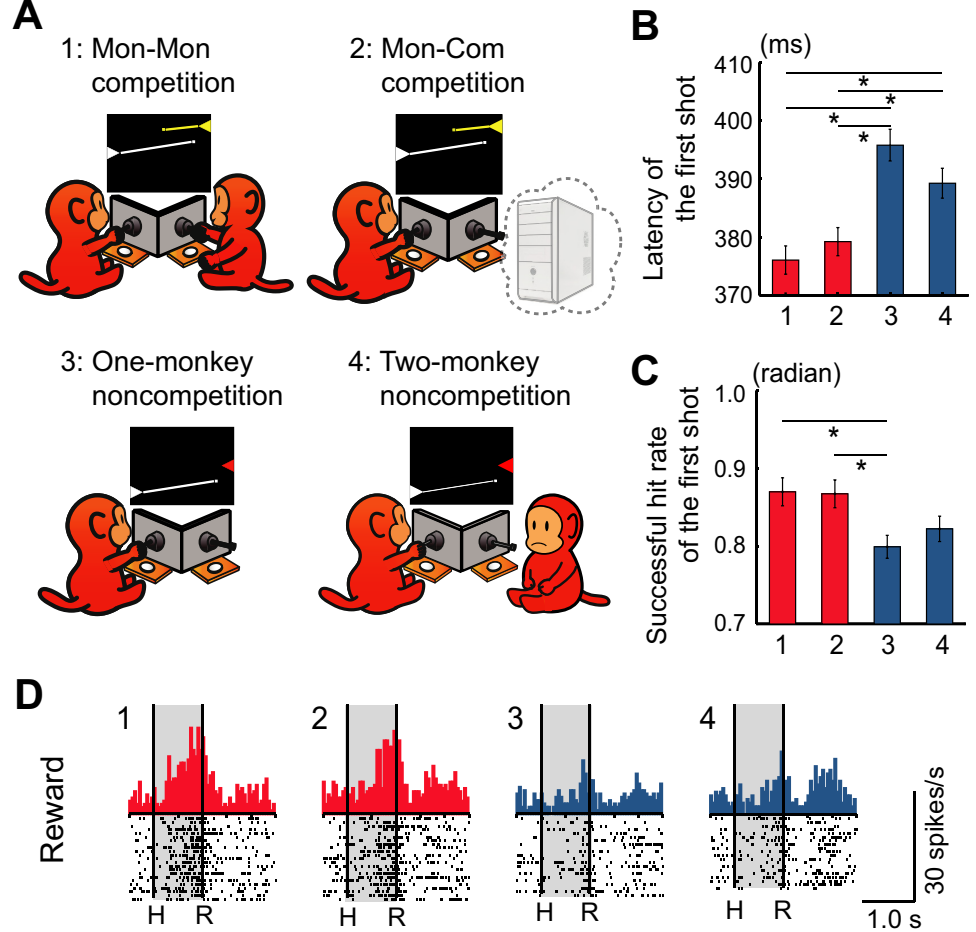

E

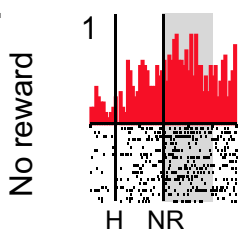

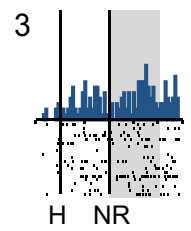

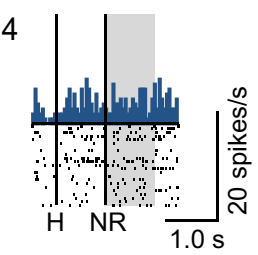

$\mathbf{F}$

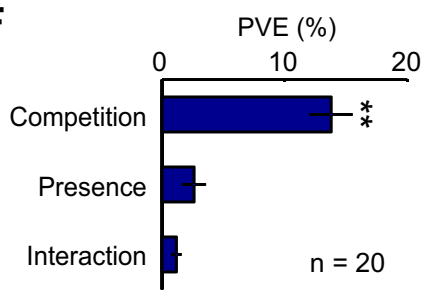

Figure 6. Effects of competition and the presence of another monkey on monkey behavior and LPFC neuronal activity. A, Schematic diagrams of the Mon-Mon competitive (1), Mon-Com competitive (2), One-monkey noncompetitive (3), and Two-monkey noncompetitive (4) conditions. In the Two-monkey noncompetitive condition, there were two monkeys in the experimental booth, but only one monkey played a noncompetitive game, whereas the other monkey did not participate in the game. $B, C$, Latencies and succesful hit rates of the first shot were compared among the four (Mon-Mon competition, Mon-Com competition, One-monkey noncompetition, and Two-monkey noncompetition) conditions. $\boldsymbol{B}$, Latency of the first shot (mean \pm SEM). One-way ANOVA demonstrated a significant difference in latency among the four conditions $\left(F_{(3,92)}=12.86, p<10^{-6}\right)$. Posthoc paired comparisons were conducted using a Bonferroni'scorrected, two-tailed $t$ test $\left({ }^{*} p<0.05\right) . n=24$ (each condition). C, Successful hit rate of the first shot (mean \pm SEM). Data were normalized by the arcsine transformation before statistical analyses. One-way ANOVA demonstrated a significant difference in successful hitrate among the four conditions $\left(F_{(3,92)}=4.29, p=0.007\right)$. Posthoc paired comparisons were conducted using a Bonferroni's-corrected, two-tailed $t$ test $\left({ }^{*} p<0.05\right) . n=24$ (each condition). $\boldsymbol{D}, \boldsymbol{E}$, Examples of neurons that showed greater activity in reward trials $(\boldsymbol{D})$ and in no-reward trials $(\boldsymbol{E})$ during the competitive compared with during the noncompetitive conditions. Neuronal activities during reward trials $(\boldsymbol{D})$ and no-reward trials $(\boldsymbol{E})$ under each condition are shown. Each shaded area indicates the period when typical activity in relation to competition and the presence of another monkey was observed. The configuration of each raster and histogram display is the same as in Figure 3A-C. For B-E: 1, Mon-Mon competition; 2, Mon-Com competition; 3, One-monkey noncompetition; 4, Two-monkey noncompetition. $F$, Mean PVE for the competitive neurons that were recorded in the four conditions. The plot shows the averaged PVE of each factor in the two-way ANCOVA (competition and presence factors and their interaction) (mean \pm SEM). We used a two-tailed Mann-Whitney $U$ test to determine whether these PVE values were significantly greater than the chance level by comparing them with those calculated from the randomized data. ${ }^{* *} p<10^{-7}$.

from the randomized data combining all the competitive and noncompetitive trials for each neuron. Only the competition factor significantly contributed to the activities of these neurons
(Mann-Whitney $U$ test, $p<10^{-7}$ ). Thus, the effect of competition on the competitive neuronal activity was more profound than the effect of the presence of another monkey, indicating that competitive LPFC neurons showed differential activities not because there was another monkey present but because the game was competitive.

\section{Discussion}

In this study, we found that monkeys' behaviors were significantly different between the competitive and noncompetitive games. The monkeys shot bullets more quickly and more accurately in the competitive than in the noncompetitive condition, indicating that they were more highly motivated in competitive than in noncompetitive games (Fig. 2). These observations are consistent with those in a previous behavioral study (Washburn et al., 1990).

Also, many LPFC neurons showed differential activity between the competitive and noncompetitive conditions in relation to the response outcome (reward and no reward) (Fig. 3). These differential LPFC activities were observed despite the similarity between the competitive and noncompetitive conditions. The amount of juice reward, the required response to get the reward (hitting the target), and the auditory and visual stimuli presented after a successful hit were identical between the competitive and noncompetitive conditions. The reward probability was also comparable between the conditions. We did not observe any difference in eye movements or in eye positions between the competitive and noncompetitive conditions.

Because LPFC is concerned with motivation (Kobayashi et al., 2002; Watanabe and Sakagami, 2007; Pan et al., 2008) and the monkey's motivation was higher in the competitive condition, it may be argued that the competitive activity simply reflects the monkey's enhanced motivational level. However, most of the competitive neurons maintained the differential activity even when we analyzed the data after eliminating the effects of the differential motivational levels on neuronal activity, suggesting that the competitive activity does not simply reflect the monkey's motivational level.

What aspect other than the motivational level induced differences in LPFC neuronal activity between the competitive and noncompetitive games? It may be the difference in response requirement to obtain a reward. The monkey had to hit the target before the rival did in the competitive but not in the noncompetitive game. In the competitive game, whether or not the monkey could obtain a reward de- 
pended not only on how well (fast and accurate) it performed the game but also on how well the rival did. This competitive nature of the games likely induced the competitive activity observed in this study.

Then, what do the competitive activities reflect? A previous study indicated that winning a competition could enhance reward value in monkeys (Washburn et al., 1990). It is also known that LPFC neurons encode reward value (Amemori and Sawaguchi, 2006b; Kim et al., 2008) and show differential activity depending on whether or not a monkey expects and/or obtains a more preferable outcome (Watanabe, 1996; Leon and Shadlen, 1999; Hikosaka and Watanabe, 2000). Another study indicated that LPFC neurons respond differently depending on whether or not the monkey anticipates the absence of a more preferable reward (Watanabe et al., 2002). Thus, Win-competitive activity may reflect the monkey's expecting/obtaining a reward whose value may have been enhanced in the competitive condition, whereas Lose-competitive activity may reflect the monkey's expecting/facing the absence of a highly valued reward during the competitive condition. Another interpretation for Wincompetitive and Lose-competitive activities is that they may code the result of a competition. A previous study reported LPFC neurons that coded the correctness of a monkey's behavior in relation to whether the behavioral response was correct or not independent of the presence or absence of a reward (Watanabe, 1989). To win a competition, it is critical to adjust behavior according to the previous response outcome (win or lose). Thus, Wincompetitive and Lose-competitive activities may play important roles in monitoring the response outcome in a competition.

Another group of neurons showed competition-induced activity changes regardless of the presence or absence of the reward (Win-Lose-competitive activity; Fig. 3C). This type of neuron may be more concerned with differentiating competitive from noncompetitive situations than with differentiating between positive and negative outcomes. Similar valenceindependent, outcome-related neuronal activities were reported previously in LPFC (Kobayashi et al., 2006). Also, previous studies reported that LPFC neurons show contextdependent activities (White and Wise, 1999; Wallis et al., 2001; Watanabe et al., 2002; Amemori and Sawaguchi, 2006a,b; IchiharaTakeda and Funahashi, 2008; Kennerley and Wallis, 2009), which are related to coding cognitive and/or motivational context information. Win-Lose-competitive activity may thus be concerned with coding the cognitive and motivational context regarding competitive situations. Competition-related differential baseline activities observed in the present study may also be concerned with monitoring the cognitive and motivational context regarding competitive situations, because previous studies reported that LPFC neurons show context-monitoring baseline activities (Watanabe et al., 2002; Amemori and Sawaguchi, 2006a,b).

LPFC neurons appear to be sensitive to social factors because we found some that showed differential activity between when the rival was another monkey and when it was the computer (Fig. 5 ). Because the mere presence of another monkey (Two-monkey noncompetition) did not induce competitive LPFC activity (Fig. 6 ), it is likely that the presence of an actual rival specifically induced the competitive activity. Although a previous study showed that the responses of LPFC neurons differed depending on the social rank of the counterpart monkey in a food-grab task (Fujii et al., 2009), the social rank between the monkeys probably did not affect neuronal activity in this study because we did not find a clear dominant-subordinate relationship between the monkeys that competed against each other in the shooting games.
However, we found differences in the monkey's behavior, as well as neuronal activity, depending on the animacy of the rival. One of the monkeys (monkey $\mathrm{H}$ ) showed better behavioral performance in the Mon-Mon competitive than in the Mon-Com competitive condition (shorter latency for the first shot and better hitting accuracy; Fig. 2). Furthermore, the monkey's licking behavior was different in lose trials between the Mon-Mon and Mon-Com competitive conditions. Although there was no difference in licking latency in win trials between the conditions, licking behavior in lose trials appeared much later in the MonMon than in the Mon-Com competition. This behavioral difference may be explained by the difference in the animal's attention between the conditions. When a monkey won a competition, it may have focused its attention on receiving the coming reward in both conditions, yielding no difference in licking latency between the two. When the monkey lost a competition, however, its attention may have been caught by the other monkey receiving a reward in the Mon-Mon competition, but not in the Mon-Com competition, in which there was no monkey that received a reward. Although the monkey could hear the sound of the solenoid valve that delivered the juice to the reservoir even in lose trials in the Mon-Com competition, it seems that the sound did not catch the monkey's attention so much. Thus, it is likely that seeing the rival receive a reward induced the delay in the onset of spontaneous licking when the monkey lost the game in the Mon-Mon competition. These results indicate that the social situation in which the rival is another monkey appears to make the monkey pay more attention to the rival than the nonsocial situation in which the rival is an inanimate computer. Attention to an animate rival may modulate neuronal activities of LPFC neurons, which may then lead to better performance in socially competitive situations. Additional studies are needed to examine the effects of social factors on LPFC neuronal activity by using various kinds of situations, such as competition between dominant and subordinate monkeys or between monkeys and humans.

It is also important to study other brain regions using the same tasks, especially the medial prefrontal cortex (MPFC) that is indicated to be involved in social functions (Decety et al., 2004; Behrens et al., 2008; Zink et al., 2008; Marsh et al., 2009; Zahn et al., 2009; Tricomi et al., 2010), and compare response properties of neurons among different regions. Indeed, recent human neuroimaging studies indicate that the MPFC, including the anterior cingulate area, plays the most important role in social cognition (Blakemore, 2008). An fMRI study indicated activation of the MPFC in relation to cooperative behavior during a game, with the activity being more enhanced when the subject played games with a human than with a computer (McCabe et al., 2001). Thus, it is likely that MPFC neurons would show differential activity depending on whether the rival is another monkey or the computer in our task.

In summary, the present study showed that monkeys were highly motivated to play a competitive video game and that competition induced greater modification in LPFC activity, especially in social situations in which there was competition between monkeys. It is important for survival in nature for animals to compete against others and obtain limited resources, such as food and mates. Our findings suggest that LPFC neurons may play an important role in adapting behavior to increase the chances of obtaining a reward in a socially interactive environment by monitoring response outcomes and cognitive/motivational context information regarding competitions. 


\section{References}

Amemori K, Sawaguchi T (2006a) Rule-dependent shifting of sensorimotor representation in the primate prefrontal cortex. Eur J Neurosci 23:1895-1909.

Amemori K, Sawaguchi T (2006b) Contrasting effects of reward expectation on sensory and motor memories in primate prefrontal neurons. Cereb Cortex 16:1002-1015.

Barraclough DJ, Conroy ML, Lee D (2004) Prefrontal cortex and decision making in a mixed-strategy game. Nat Neurosci 7:404-410.

Behrens TE, Hunt LT, Woolrich MW, Rushworth MF (2008) Associative learning of social value. Nature 456:245-249.

Blakemore SJ (2008) The social brain in adolescence. Nat Rev Neurosci 9:267-277.

Decety J, Jackson PL, Sommerville JA, Chaminade T, Meltzoff AN (2004) The neural bases of cooperation and competition: an fMRI investigation. Neuroimage 23:744-751.

Dorris MC, Glimcher PW (2004) Activity in posterior parietal cortex is correlated with the relative subjective desirability of action. Neuron 44:365-378.

Fujii N, Hihara S, Nagasaka Y, Iriki A (2009) Social state representation in prefrontal cortex. Soc Neurosci 4:73-84.

Fukui H, Murai T, Shinozaki J, Aso T, Fukuyama H, Hayashi T, Hanakawa T (2006) The neural basis of social tactics: an fMRI study. Neuroimage 32:913-920.

Gallagher HL, Jack AI, Roepstorff A, Frith CD (2002) Imaging the intentional stance in a competitive game. Neuroimage 16:814-821.

Garcia SM, Tor A, Gonzalez R (2006) Ranks and rivals: a theory of competition. Pers Soc Psychol Bull 32:970-982.

Hikosaka K, Watanabe M (2000) Delay activity of orbital and lateral prefrontal neurons of the monkey varying with different rewards. Cereb Cortex 10:263-271.

Ichihara-Takeda S, Funahashi S (2008) Activity of primate orbitofrontal and dorsolateral prefrontal neurons: effect of reward schedule on taskrelated activity. J Cogn Neurosci 20:563-579.

Kennerley SW, Wallis JD (2009) Reward-dependent modulation of working memory in lateral prefrontal cortex. J Neurosci 29:3259-3270.

Kim S, Hwang J, Lee D (2008) Prefrontal coding of temporally discounted values during intertemporal choice. Neuron 59:161-172.

Kobayashi S, Lauwereyns J, Koizumi M, Sakagami M, Hikosaka O (2002) Influence of reward expectation on visuospatial processing in macaque lateral prefrontal cortex. J Neurophysiol 87:1488-1498.

Kobayashi S, Nomoto K, Watanabe M, Hikosaka O, Schultz W, Sakagami M (2006) Influences of rewarding and aversive outcomes on activity in macaque lateral prefrontal cortex. Neuron 51:861-870.

Leon MI, Shadlen MN (1999) Effect of expected reward magnitude on the response of neurons in the dorsolateral prefrontal cortex of the macaque. Neuron 24:415-425.

Marsh AA, Blair KS, Jones MM, Soliman N, Blair RJ (2009) Dominance and submission: the ventrolateral prefrontal cortex and responses to status cues. J Cogn Neurosci 21:713-724.

McCabe K, Houser D, Ryan L, Smith V, Trouard T (2001) A functional imaging study of cooperation in two-person reciprocal exchange. Proc Natl Acad Sci U S A 98:11832-11835.

Pan X, Sawa K, Tsuda I, Tsukada M, Sakagami M (2008) Reward prediction based on stimulus categorization in primate lateral prefrontal cortex. Nat Neurosci 11:703-712.

Reeve J, Olson BC, Cole SG (1985) Motivation and performance: two consequences of winning and losing in competition. Motiv Emot 9:291-298.

Rilling JK, Sanfey AG, Aronson JA, Nystrom LE, Cohen JD (2004) The neural correlates of theory of mind within interpersonal interactions. Neuroimage 22:1694-1703.

Rumbaugh DM, Richardson WK, Washburn DA, Savage-Rumbaugh ES, Hopkins WD (1989) Rhesus monkeys (Macaca mulatta), video tasks, and implications for stimulus-response spatial contiguity. J Comp Psychol 103:32-38.

Sanfey AG, Rilling JK, Aronson JA, Nystrom LE, Cohen JD (2003) The neural basis of economic decision-making in the Ultimatum Game. Science 300:1755-1758.

Seo H, Lee D (2007) Temporal filtering of reward signals in the dorsal anterior cingulate cortex during a mixed-strategy game. J Neurosci 27:8366-8377.

Thevarajah D, Mikulić A, Dorris MC (2009) Role of the superior colliculus in choosing mixed-strategy saccades. J Neurosci 29:1998-2008.

Tricomi E, Rangel A, Camerer CF, O’Doherty JP (2010) Neural evidence for inequality-averse social preferences. Nature 463:1089-1091.

Wallis JD, Anderson KC, Miller EK (2001) Single neurons in prefrontal cortex encode abstract rules. Nature 411:953-956.

Washburn DA, Hopkins WD, Rumbaugh DM (1990) Effects of competition on video-task performance in monkeys (Macaca mulatta). J Comp Psychol 104:115-121.

Watanabe M (1989) The appropriateness of behavioral responses coded in post-trial activity of primate prefrontal units. Neurosci Lett 101:113-117.

Watanabe M (1996) Reward expectancy in primate prefrontal neurons. Nature 382:629-632.

Watanabe M, Sakagami M (2007) Integration of cognitive and motivational context information in the primate prefrontal cortex. Cereb Cortex 17 [Suppl 1]:i101-i109.

Watanabe M, Hikosaka K, Sakagami M, Shirakawa S (2002) Coding and monitoring of motivational context in the primate prefrontal cortex. J Neurosci 22:2391-2400.

White IM, Wise SP (1999) Rule-dependent neuronal activity in the prefrontal cortex. Exp Brain Res 126:315-335.

Zahn R, Moll J, Paiva M, Garrido G, Krueger F, Huey ED, Grafman J (2009) The neural basis of human social values: evidence from functional MRI. Cereb Cortex 19:276-283.

Zink CF, Tong Y, Chen Q, Bassett DS, Stein JL, Meyer-Lindenberg A (2008) Know your place: neural processing of social hierarchy in humans. Neuron 58:273-283. 